\title{
Surgical site, renal fossa and vena caval recurrence of renal cell carcinoma: an unusual case of late recurrence
}

\author{
L. N. Seneviratne, J. M. N. R. K. Jayasundare and N. D. Perera \\ Department of Urology, National Hospital of Sri Lanka, Colombo, Sri Lanka.
}

\begin{abstract}
A 57-year old male who had undergone a radical nephrectomy for T2NoMo Fuhrman grade III tumour 5 years back developed persistent loin pain. Clinically he had a palpable right hypochondriac mass. Imaging revealed a tumour over the previous surgical scar, renal fossa with inferior vena cava obstruction with no other metastatic disease. He underwent excision of the local recurrence, cavotomy, thrombectomy with caval resection since the tumour was adherent to the IVC. Histology confirmed it as a recurrence of renal cell carcinoma and was followed by targeted therapy.
\end{abstract}

We report an unusual case of late presentation of surgical site and renal fossa recurrence with invasion to the inferior vena cava (IVC) in a patient who underwent radical nephrectomy for a renal cell carcinoma (RCC). A review of the literature showed no previous published

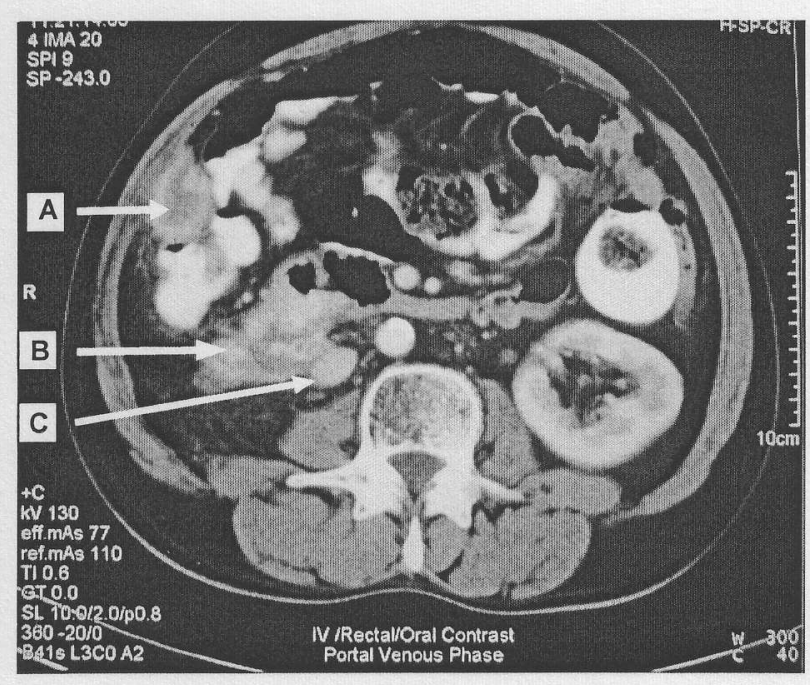

Figure 1. CT scan of the abdomen.
$A$ - Tumour recurrence at the surgical site
B - Renal fossa tumour recurrence
C - Tumour in the inferior vena cava

cases of RCC recurring in the surgical site, renal fossa with IVC involvement in combination in a single patient.

\section{Case}

A 57-year old man who had undergone a radical nephrectomy (T2NoMo grade III) 5 years back presented with a right hypochondriac mass. Computer tomography (Figure 1) and MRI (Figure 2) scan identified a mass attached to the surgical scar, in the renal fossa with invasion and near total occlusion of the IVC. Bone scan and CT chest were negative. Excision of the recurrence, cavotomy and thrombectomy with caval resection were successfully performed. Histology confirmed tumour as recurrence of renal cell carcinoma. Thereafter he was followed up with targeted therapy.

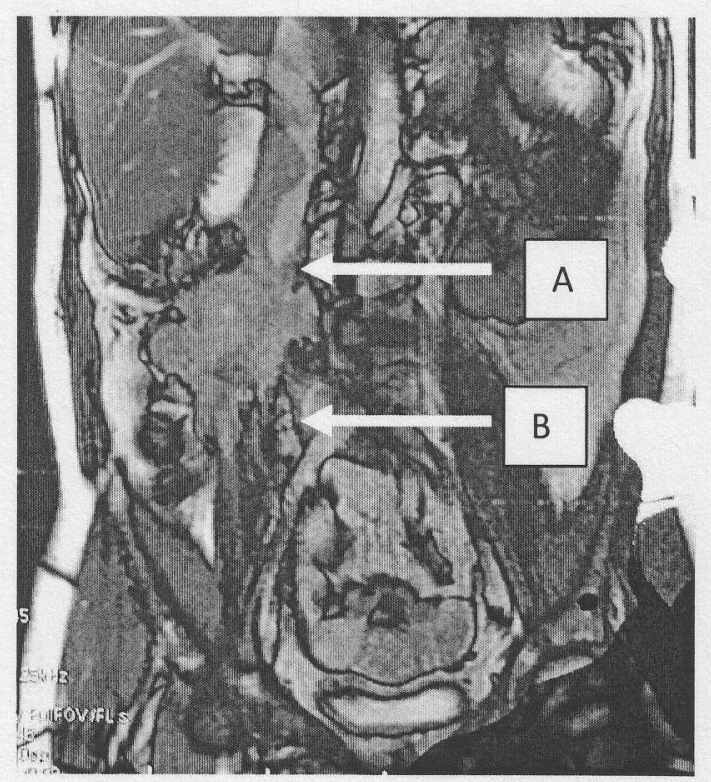

Figure 2. Vertical reconstruction film of MRI scan.

A - Tumour thrombus in the IVC

B - Renal fossa recurrence tumour infiltrating the IVC 


\section{Discussion}

Tumour recurrence of RCC reported varies from $10-37 \%$ where metastatic recurrence predominates with lung (63\%), bone (27\%), brain (20\%), liver (16\%), retroperitoneal lymph nodes (10\%) far being the common sites(1). Local recurrence of RCC after radical nephrectomy remains an uncommon event. Published series report that between $2 \%$ and $5 \%$ of patients with RCC will develop a local recurrence after radical nephrectomy (2). However, majority of patients with local recurrence also has systemic disease at diagnosis; only $10-17 \%$ of patients have true isolated local recurrences.

Time to tumour recurrence generally occurs within the first 2 years with generally $83 \%$ affected (3). Though late metastatic recurrence is reported even after 20 years, literature on late local recurrence is scarce (4). The mean time of tumor recurrence usually is 17 months (range, 3-50 months).

Few pathological features have been proposed as risk factors that predict an increased risk of local recurrence of the disease. These include tumour size $>5 \mathrm{~cm}, \mathrm{~T}$ stage $>$ T3a, T3b, Robson stage III or more, histological grade 3,4 or node-positive disease in some but not all (5).

Not all patients with locally recurrent disease will be symptomatic at presentation. The presence of symptoms leading to the discovery of a recurrence varies widely from $7 \%$ to $73 \%$. The majority of the published series shows that patients who died had a mean time to recurrence of 16 months, compared with 79 months for patients who survived. These findings reinforce the well-known heterogeneity of the characteristics of RCC and its clinical behaviour (6).

Since RCC has poor response to adjuvant treatments, surgery is the best choice in a case of local recurrence or isolated metastatic recurrence in the absence of metastatic disease in other places. An aggressive surgical resection can provide up to 30\% 5-year disease-free survival. However, complete surgical resection with negative surgical margins is crucial and en bloc resection of adjacent organs should be done to achieve this goal. Failing to do so will likely result in recurrent disease and decreased survival. It is not surprising, then, that most published series report complication rates ranging from $18 \%$ to $47 \%$ (7).

Surgery for IVC recurrence is rarity. If encountered the options available are tumor thrombus removal and primary cavotomy closure, IVC ligation and removal of tumour thrombus without vascular reconstruction and IVC wall excision and placement of a bovine pericardium or PTFE graft (8). Although technically difficult, resection of IVC tumour and primary closure as in this case is an acceptable method of treatment.

Although many of the published reports support the potential benefit associated with an aggressive surgical approach, the role of systemic therapy or radiation therapy remains to be defined. So far, most authors report that disease control with both treatment modalities is far more less over surgery. Radiation therapy may be of value for palliation of symptomatic local recurrence for patients who are not surgical candidates.

Prognosis of these patients with recurrence has an inverse correlation between the number of risk factors which include time from diagnosis to start of systemic therapy $<12$ months, Karnofsky index $<80 \%$, serum corrected calcium $>10 \mathrm{mg} / \mathrm{dL}$, haemoglobin less than the age/sex-specific lower limit of normal and an LDH more than $1.5 \times$ the upper limit of normal. Patients were categorized into those with low risk (no risk factors), intermediate risk (1-2 risk factors present) and high risk (3-5 risk factors present) 2-year overall survival rates were $88 \%, 51 \%$ and $11 \%$, respectively (5).

We emphasize the need for life long strict surveillance for all patients with RCC for early diagnosis of this rare recurrence involving specially the IVC so that surgery becomes a viable option of treatment.

\section{References}

1. Eun Jin C. Renal cell carcinoma: Analysis of postoperative recurrence patterns. Radiology 2005; 234: 189.

2. Schrodter S et al. Outcome of surgical treatment of isolated local recurrence after radical nephrectomy for renal cell carcinoma. Journal of Urology 2002; 167: 1630-3.

3. Nyree G, Martin EG, Aslam S. Imaging in metastatic renal cell carcinoma. American Journal of Radiology 2007; 189: 360-70.

4. Featherstone JM, Bass P, Cumming J, Smart CJ. Solitary, late metastatic recurrence of renal cell carcinoma: Two extraordinary cases. International Journal of Urology 13; 12: 1525-7.

5. Eggener SE et al. Renal cell carcinoma recurrence after nephrectomy for localized disease: Predicting survival from time of recurrence. Journal of Clinical Oncology 2006 Jul 1; 24(19): 3101-6.

6. Master VA et al. Management of isolated renal fossa recurrence following radical nephrectomy. Journal of Urology 2005; 174: 473-7. 
7. Bruno JJ et al. Renal cell carcinoma local recurrences: Impact of surgical treatment and concomitant metastasis on survival. British Journal of Urology International 2006; 97: 9338.
8. Smaldone MC, Cannon GM, Hrebinko RL. Resection of recurrent inferior vena cava tumor after radical nephrectomy for renal cell carcinoma. Urology 2006; 67(5): 1084e4-1084e7.

\section{Authors}

L. Niroshan Seneviratne, MS (Col), MRCS (Eng)*

J. M. N. R. K. Jayasundare, MS (Col)*

*Senior Registrar in Urology

Neville D. Perera, MS (Col), FRCS (Eng), FRCS (Edin), DUrol (Lond)

Consultant Urological Surgeon

Department of Urology, National Hospital of Sri Lanka, Colombo, Sri Lanka 